\title{
Characterization of Propellant Flow and Bias Required to Initiate an Arc Discharge in a Heaterless Hollow Cathode
}

\author{
Ryan K. Ham, ${ }^{1}$ and John D. Williams ${ }^{2,3}$ \\ Colorado State University, Fort Collins, CO, 80523, USA \\ Scott J. Hall ${ }^{4}$ \\ Vantage Partners, LLC, NASA Glenn Research Center, Cleveland, OH, 44135, USA \\ and \\ Gabriel F. Benavides, ${ }^{5}$ and Timothy R. Verhey ${ }^{6}$ \\ NASA Glenn Research Center, Cleveland, OH, 44135, USA
}

\begin{abstract}
Heaterless hollow cathodes provide an opportunity to reduce complexity and improve reliability in electric propulsion systems. While removal of the heater has little effect on steady-state operation of a hollow cathode, it has a considerable effect on the ignition process. To successfully integrate a heaterless hollow cathode into a spaceflight electric propulsion system, it will be necessary to establish definitive requirements for the propellant feed and electrical subsystems so that ignition of a plasma discharge can be achieved reliably. The aim of this research was to form a better understanding of these requirements by performing an investigation of the propellant flow and voltage conditions required for the ignition of a plasma arc discharge. This aim was achieved by performing discharge initiation experiments using both a specially designed experimental apparatus and a functional heaterless hollow cathode assembly. It was demonstrated that there is a distinct difference in the voltage required to initiate a plasma discharge between two common electric propulsion propellants, xenon and krypton, which suggests that the developmental testing of heaterless hollow cathodes needs to be performed with the appropriate propellant gas species. Heaterless hollow cathode ignition experiments showed that the keeper orifice diameter has a strong effect on the voltage required to ignite a plasma discharge at a given propellant mass flow rate, while the effect of keeper-cathode separation distance was only strong at flow rates below $25 \mathrm{sccm}(\mathrm{Xe})$.
\end{abstract}

\section{Introduction}

Hollow cathode heaters are required to perform many thousands of heating cycles without degradation in performance. They are difficult to design and manufacture, and due to delicate features, they have been shown to exhibit highly variable lifetimes when subject to thermal cycle lifetime testing [1,2]. Because failure of the cathode heater presents a single-point failure mechanism of a thruster, evaluation of the performance and lifetime of cathode heaters is necessary. This can be a costly and time-consuming process in the flight qualification of a hollow cathode assembly. Furthermore, cathode heaters can impose undesirable volume and power requirements, especially when considering smaller diameter hollow cathodes for small, low-power Hall-effect thrusters, which are currently being developed to provide propulsion for small satellites. Heaterless hollow cathodes provide an opportunity to improve the reliability and simplify electric propulsion thrusters by eliminating the need for a cathode heater. While heaterless hollow cathodes provide several distinct advantages, there are concerns that the lack of a heater will prevent conditioning of the cathode insert, complicate the ignition process, and introduce undesirable complexities in the electrical and feed systems. Before considering heaterless hollow cathodes for space flight applications, it is necessary

${ }^{1}$ Graduate Research Assistant, Department of Mechanical Engineering.

${ }^{2}$ Associate Professor, Department of Mechanical Engineering.

${ }^{3}$ Technical Director, Plasma Controls, LLC.

${ }^{4}$ Research Engineer, Electric Propulsion Systems Branch, Member AIAA.

${ }^{5}$ Research Engineer, Electric Propulsion Systems Branch, Member AIAA.

${ }^{6}$ Senior Research Engineer, Electric Propulsion Systems Branch, Member AIAA. 
that the ignition process be well understood, and that electrical and feed system requirements be determined. To develop a better understanding of the ignition process of heaterless hollow cathodes, a study was performed in which ignition behavior was evaluated for a range of propellant gas pressure and bias conditions.

Hollow cathodes utilize an insert formed from a low work-function emitter material that allows the cathode to operate at moderate temperatures. These cathodes have demonstrated lifetimes in the tens-of-thousands of hours [35]. Traditional hollow cathodes equipped with a heater can be "conditioned", a process in which impurities such as $\mathrm{CO}_{2}$ and $\mathrm{H}_{2} \mathrm{O}$ that may have bonded with emitter materials during exposure to atmosphere are driven out during a gradual heating process [6]. The heater is also used to raise the temperature of the cathode close to its operating temperature $\left(1100{ }^{\circ} \mathrm{C}\right.$ or more) prior to ignition. When the cathode emitter is heated to these temperatures, thermionic emission of electrons at moderate current becomes possible. By applying a voltage between the cathode and the nearby keeper electrode, the electrons emitted by the cathode are accelerated towards the keeper. When propellant gas is flowing through the hollow cathode assembly, the accelerating electrons collide with neutral atoms causing a chain reaction of ionization events, and the rapid formation of a plasma arc discharge. This ignition process, when attempted with a cold cathode (as in a heaterless hollow cathode), has been shown to require higher voltage and elevated propellant flow rates due to the lack of thermionic electron emission from the cathode at the beginning of the ignition process [7-10]. After ignition, the cathode is heated by ions from the plasma arc discharge and the cathode temperature rises until thermionic emission begins to become a significant fraction of the discharge current, at which point the propellant flow rate may be reduced to nominal levels. Also at this point in the ignition process, the electrode voltages drop to values similar to what are observed with a traditional hollow cathode equipped with a heater. The processes of cathode conditioning and ignition are the only fundamental differences between traditional cathodes and heaterless cathodes. So far, heaterless hollow cathodes have not been used in space flight applications, which is likely a result of the lack of understanding of the ignition process and the lack of the existence of $\mathrm{BaO}$-based emitters that do not require conditioning. To integrate a heaterless hollow cathode in a modern satellite propulsion system, it will be necessary to have an adequate understanding of the propellant and electrical requirements needed for ignition, the long term effects of no conditioning, and the long term effects of many thousands of arc ignition events. An investigation of the propellant and electrical requirements was carried out by performing two distinct experiments: (1) discharge initiation in a specially designed apparatus that employed a well-defined electrode separation distance in a parallel plate configuration at uniform and controlled propellant pressures, and (2) discharge initiation in a small, low-power heaterless hollow cathode using an enclosed keeper configuration.

\section{Facilities and Equipment}

Vacuum facilities at two separate laboratories were used in this research. Vacuum Facility 17 (VF-17) at NASA Glenn Research Center is a cylindrical vacuum chamber with a diameter of $0.9 \mathrm{~m}$ and a length of $2.1 \mathrm{~m}$. VF-17 is equipped with a cryogenic pump which has demonstrated a pumping speed of approximately $2000 \mathrm{~L} / \mathrm{s}$ of xenon at room temperature [11]. At Colorado State University, a similar cylindrical vacuum chamber was used which is $1.0 \mathrm{~m}$ in diameter and $1.9 \mathrm{~m}$ in length and equipped with a turbomolecular pump capable of pumping xenon at a speed of approximately $1800 \mathrm{~L} / \mathrm{s}$. Each facility was outfitted with a laboratory gas feed system that utilized $50 \mathrm{sccm}$ mass flow controllers. To supply the bias required to perform ignition testing, high-voltage DC power supplies were used. The power supply used in the testing at NASA Glenn Research Center was a $1000 \mathrm{~V}$ capable supply, while the power supply used at Colorado State University was a $600 \mathrm{~V}$ capable supply. In both cases, the leads from the power supply output were routed to high-voltage feedthroughs on the vacuum chamber and then to the cathode and anode/keeper within the chamber. The electrodes were at a floating potential with respect to ground to avoid electrical interaction with the facility. To monitor and record the electrical behavior between electrodes during testing, an oscilloscope was used to sense both the voltage and the current flow and log these data at a high sampling rate. A $100 \Omega$ ballast resistance was placed in series with the electrical load imposed by the keeper-cathode discharge to limit the peak current during ignition experiments. With these two similar setups, heaterless hollow cathode ignition experiments could be

performed across a broad range of conditions. Experiments that were performed at both facilities showed similar results.

\section{A. Discharge Characterization Unit}

To investigate the conditions required to initiate the formation of a plasma discharge, a discharge characterization unit was developed. A cross-section view of this device is shown in Fig. 1. The device was situated within a vacuum chamber so that gaseous impurities could be avoided and also so that breakdown events along the exterior of the device could be prevented. The discharge characterization unit was positioned at the end of a propellant feed system so that high purity propellant gas could be flowed into the device at a controlled rate. By placing electronically 
controlled solenoid valves at the upstream and downstream ends of the device, an arbitrary pressure of propellant gas could be established and maintained between the electrodes. A gas line stemming from just upstream of the device was routed to the exterior of the vacuum chamber to a capacitance manometer so that the static pressure within the cavity could be measured. In this arrangement, the distance and gas pressure between the electrodes was uniform during each discharge initiation test and these parameters were either known or could be measured. This arrangement also allowed for various propellant gasses and/or electrode materials to be tested with minimal reconfiguration.

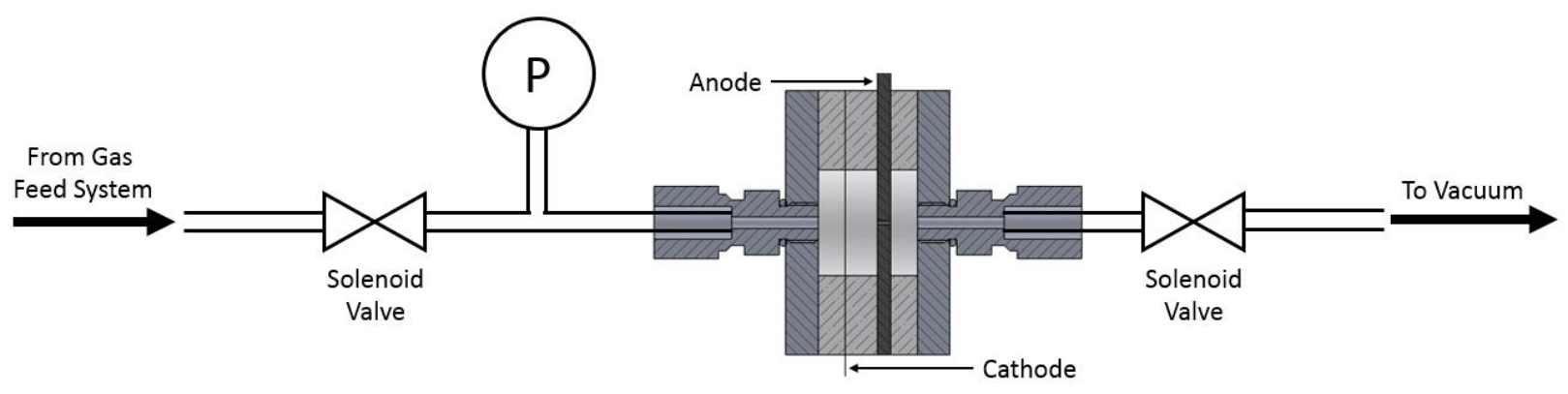

Fig. 1 Flow schematic and cross-section view of the discharge characterization unit that was used to determine breakdown voltage at a range of internal gas pressures.

\section{B. Heaterless Hollow Cathode Test Articles}

Heaterless hollow cathode ignition experiments were performed at both NASA Glenn Research Center and at Colorado State University using separate, identical hollow cathode assemblies. The heaterless hollow cathodes assemblies were provided by Plasma Controls, LLC [12]. A $3.2 \mathrm{~mm}$ diameter cathode was selected because it is of a suitable size for low power Hall-effect thrusters; a thruster class that could be vastly improved by integrating heaterless hollow cathode technology. The cathode consisted of a tantalum tube with a formed spherical tip within which a porous tungsten barium oxide emitter was housed. The cathode was mounted within a hollow cathode assembly that utilized an enclosed keeper, which was specially designed to be an experimental evaluation unit. Using this assembly, shown in Fig. 2, it was possible to adjust the axial position of the cathode relative to the keeper, and the keeper orifice plate could be easily removed and replaced with a different keeper orifice plate geometry. The keeper was made from graphite and designed with a slender profile so that the assembly could be integrated into a low power Hall-effect thruster. In another study, the heaterless hollow cathode was operated in a steady-state manner at discharge currents up to $3 \mathrm{~A}$.

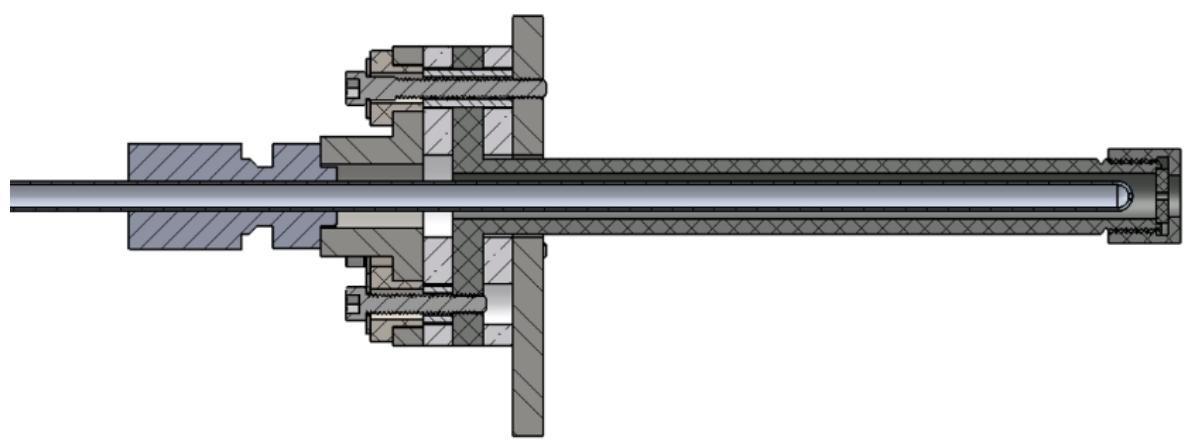

Fig. 2 Cross-section view of the heaterless hollow cathode that was used in this study (insert not shown). The enclosed graphite keeper structure includes features at the downstream end that allow for the orifice plate to be removed and replaced.

\section{Conditions Required to Initiate a Plasma Discharge}

Because the ignition process in a heaterless hollow cathode begins with the electrical breakdown of propellant gas, (i.e. the formation of a plasma discharge), a study was performed to determine the conditions that are necessary for this phenomenon to occur. The electrical breakdown of a gas is a process in which free electrons are accelerated by an electric field that is formed between two electrodes. Electrons that gain sufficient kinetic energy can (upon colliding 
with a neutral atom) cause an ionization event. It is necessary that certain conditions are met such that this ionization process will occur in a runaway fashion and result in the formation of a plasma discharge. These conditions are dependent upon the strength of the electric field, the secondary electron yield of the cathode material, the electron mean free path, and the ionization cross section and ionization energy threshold of the gas. Paschen's Law incorporates these conditions into an equation that shows that the voltage required to initiate a plasma discharge in a given gas species is a function of the product of the gas pressure and the distance between electrodes [13, 14]. A plot of the breakdown voltage and pressure-distance product relationship is called a Paschen curve. It is a useful tool when investigating the flow rate and bias voltage required to ignite a plasma discharge in a heaterless hollow cathode because the gas pressure between the electrodes in a hollow cathode can be assumed to be related to the propellant mass flow rate. While Paschen curves have been presented in a number of studies, most of these data are not directly applicable to electric propulsion hollow cathodes due to the materials and/or gasses used. In this study, several Paschen curves were experimentally measured using various electrode materials and electric propulsion propellants. The experimental procedure and results are detailed in this section.

\section{A. Procedure for Determining Breakdown Voltage as a Function of Internal Pressure}

This experiment was performed using the discharge characterization unit described in section II, within which a static, uniform pressure of propellant gas was established between two parallel, planar electrodes. To develop Paschen curves for various propellant species and cathode materials, it was necessary to contain a known pressure of highpurity gas within the discharge characterization unit, and then to determine the breakdown voltage of the gas in this state. This was accomplished with high repeatability and minimal uncertainty using the following steps. First, before performing a test, a bypass valve was opened to the vacuum chamber, and the feed system (along with the interior of the device) was evacuated for a minimum of 12 hours to remove impurities in the form of residual gasses from the experiment. Afterwards, using the laboratory gas feed system, propellant gas was flowed into the device where it was held at a static pressure by closing the solenoid valves at the upstream and downstream ends of the device. A slowly increasing bias was then applied between the electrodes until an electrical breakdown of the gas was observed. A breakdown event was sensed by the oscilloscope as a flow of current between the electrodes. At the moment that a breakdown event was observed, the bias and the gas pressure between the electrodes was recorded, and upon detection of the formation of a plasma discharge, the power supply was immediately switched off to avoid excessive heating of the electrode materials. After acquiring breakdown voltage data over a range of internal pressures, a Paschen curve was formed by plotting the breakdown voltage as a function of the product of the pressure and distance between electrodes. Given the parallel plate electrode configuration in the discharge characterization unit, the distance between the electrodes was equal to the thickness of the dielectric spacer that was used to offset the two electrodes. After each test sequence, the propellant gas species could be changed, and/or the device could be reassembled with different electrode materials.

\section{B. Results of the Discharge Initiation Study}

For this experiment, a breakdown event was defined as the formation of a glow or an arc discharge. A glow discharge was evidenced by a current flow of several milliamps, whereas the formation of an arc discharge was evidenced by the power supply switching to current limited operation at the setting of 0.5 A. Formation of an arc discharge was generally observed at higher internal pressures. At lower internal pressures, glow discharges were more commonly observed. The cause of this disparity will be investigated in a follow-on study, which will aim to form a distinction between the conditions that result in the formation of a glow discharge, rather than the formation of an arc discharge. Because this experiment was performed with the intent to develop Paschen curves, glow discharge and arc discharge events were treated equally and both were characterized as breakdown events.

Given the layout of the discharge characterization unit, it is possible that the dielectric spacer located between the cathode and anode electrodes could have enhanced the production of free electrons under certain electric field conditions. This would have occurred at the triple junction (metal-insulator-gas interface) formed where the conductive cathode and the dielectric spacer meet within the gaseous interior of the device. It is theorized that electric field enhancement can occur at the triple junction due to polarization of the dielectric and subsequent increase of surface charge density in the conductor near the location of the triple junction. Furthermore, it is possible that secondary electron emission could occur at the dielectric surface, which would further enhance the electric field at the triple junction $[15,16]$. It is not possible at the present time to predict the extent by which the triple junction effect may have impacted the results of this study.

Data were acquired for two common propellant gas species, xenon and krypton, while the device was configured with a tantalum cathode and a graphite anode (materials commonly used in modern hollow cathode assemblies) with a spacing of approximately $6.4 \mathrm{~mm}$. As shown in Fig. 3 (left), significant differences in breakdown voltage were 
observed for the different propellant gas species over a wide range of internal pressures. Specifically, krypton is shown to breakdown at significantly lower voltages than xenon. This result aligns with the theory of Paschen's Law which asserts that the ionization cross section and ionization energy threshold of the gas play an important role in the breakdown process. Another factor that affects the shape of a Paschen curve and the location of its minimum, is the secondary electron yield of the cathode material $[13,14]$. To gauge the significance of this factor, three different cathode materials were tested; tantalum (as-received), molybdenum (as-received), and tantalum that was covered with a thin dielectric film. It has been shown that a thin dielectric film upon a metallic material may cause the secondary electron yield of the material to increase by up to three orders of magnitude. This has been attributed to quantum tunneling and/or field enhancement effects [17]. If the secondary electron yield were affected to this degree, a significant change in the Paschen curve would be observed. To test this theory, a coating of titanium dioxide $\left(\mathrm{TiO}_{2}\right)$ was applied to a planar tantalum electrode with a thickness of approximately $10 \mathrm{~nm}$ using an ion sputter deposition system. Fig. 3 (right) shows the Paschen curves for xenon using the three different cathode materials. While there may be subtle differences in each curve, it is difficult to conclude that a significant difference was observed. From these data, it can be concluded that the characteristics of the gas species has the most significant effect on the Paschen curve. The data shown in Fig. 3 indicate that a heaterless hollow cathode would likely demonstrate vastly different ignition performance when operated with different propellant gas species. In general, these results show that for a given gas species and electrode spacing, there exists an optimal pressure at which a plasma discharge can be formed with a minimal voltage.
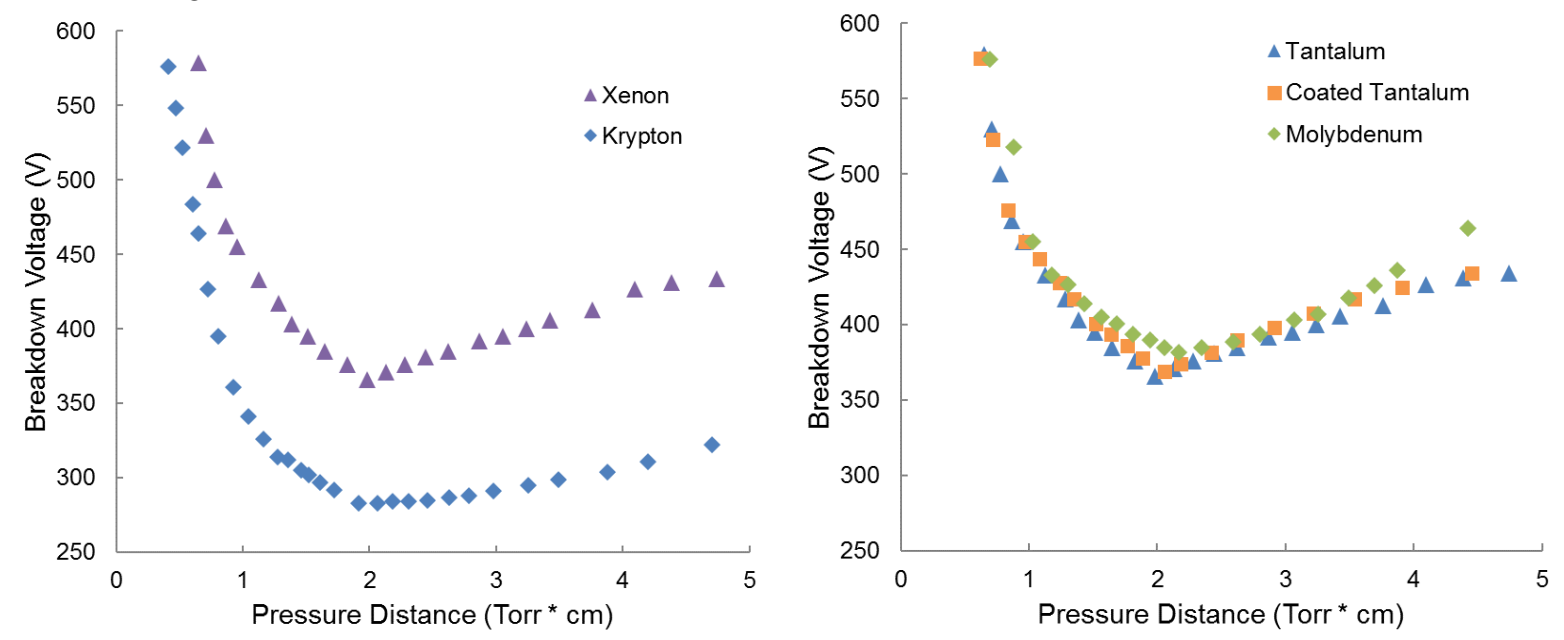

Fig. 3 Experimentally measured Paschen curves for different propellant gas species and cathode materials.

\section{Heaterless Hollow Cathode Ignition Testing}

In designing a heaterless hollow cathode, it is important to consider that exceedingly demanding ignition requirements could be problematic for electric propulsion systems that have limited propellant flow and/or electrical capability. As was shown by the above described discharge ignition experiment, both the pressure and distance between electrodes have a direct effect on the voltage required to initiate a plasma discharge. Knowledge of this relationship is useful because it shows that a minimal ignition voltage can be attained in a heaterless hollow cathode assembly by creating an optimal propellant gas pressure and distance between electrodes. Despite the well-defined Paschen theory, it is difficult to predict the ignition voltage in a hollow cathode assembly at a given flow rate because the distance between electrodes is typically non-uniform and the pressure between the electrodes varies with position and is difficult to model. In this study, a qualitative approach was taken in which two critical dimensions were investigated; the keeper orifice diameter and the distance between the cathode orifice and keeper orifice. The heaterless hollow cathode described in section II was used for this experiment. The keeper orifice diameter was considered a critical dimension because it was assumed that this would have a direct effect on the propellant gas pressure between the cathode and keeper electrodes, with smaller keeper orifice diameters causing higher pressures between the cathode and keeper electrodes. The distance between the cathode tip and keeper orifice was also considered a critical dimension because electrode spacing is a significant variable in Paschen's Law. It is worth noting that the radial distance between the outer wall of the cathode tube and the inner diameter of the keeper tube was approximately $1 \mathrm{~mm}$ and this dimension could not be altered. To determine whether these geometric parameters affect the ignition process, several hollow cathode configurations were tested and compared. 


\section{A. Procedure for Determining Ignition Voltage as a Function of Propellant Mass Flow Rate}

The purpose of this experiment was to see how the ignition performance would be impacted by varying the keeper orifice diameter or the axial distance between the cathode and keeper. To make qualitative comparisons, each geometric configuration was tested to determine the ignition voltage as a function of propellant mass flow rate. Before each test sequence, the propellant feed system was exposed to vacuum for a minimum of 12 hours to ensure that gaseous impurity concentrations would be minimized during the experiment. The first step of the test sequence was to establish a propellant mass flow rate through the hollow cathode. The flow rate was held constant by a thermal mass flow controller. To verify that the flow rate through the cathode was equal to the flow rate through the mass flow controller, the vacuum chamber pressure and the pressure upstream of the hollow cathode were monitored until stable values were reached. Then, a slowly increasing bias was applied between the cathode and keeper electrodes until the ignition of an arc discharge was observed. This could be most easily confirmed by viewing the display of the oscilloscope. In some flow conditions, a several milliamp glow discharge would be formed at a bias approximately $50 \mathrm{~V}$ less than the ignition voltage. Similar to what was observed in the discharge characterization unit, this would occur more often at lower propellant mass flow rates, and we have plans to investigate this further in a future study. For the present study, ignition was defined as the transition to a stable $1.5 \mathrm{~A}$ discharge between the cathode and keeper electrodes. After each ignition test, the voltage at which the arc discharge was initiated would be recorded. Following a successful ignition, the discharge would be extinguished within 1 second after initiation so that the cathode would not be excessively heated. Using an optical pyrometer, it was observed that the cathode tip would fall below visibly incandescent temperatures $\left(<700^{\circ} \mathrm{C}\right)$ nearly instantly after the discharge was extinguished, indicating cathode temperatures far below steady-state operating temperature. On the contrary, when extinguishing the discharge in the same hollow cathode assembly at a nominal operating temperate $\left(1150{ }^{\circ} \mathrm{C}\right)$, the tip would remain visibly incandescent for approximately 5 seconds after being switched off. To further ensure that all parts of the cathode remained at temperatures well below thermionic emission temperatures, at least 60 seconds were allowed to pass between each ignition test. A minimum of five ignition trials were performed at each flow condition. The ignition behavior showed to be repeatable with only small deviations in ignition voltage in repeated tests.

\section{B. Results}

The voltage and current between the cathode and keeper electrodes during a typical ignition event are shown in Fig. 4 (top). The ignition of a plasma arc discharge is accompanied by a dramatic drop in voltage and a simultaneous surge in current between the electrodes. The peak value of the current spike is a function of the ignition voltage and the resistance of the circuit. To avoid potentially damaging high currents, the magnitude of the current spike was limited by adding a $100 \Omega$ ballast resistance to the keeper circuit, which resulted in a current spike peak values close to the ignition voltage divided by $100 \Omega$. The exponential decay of the current spike is characteristic of a resistorcapacitor circuit in which the time constant is the product of the resistance and capacitance. In this case, the capacitance is provided by the power supply's output capacitor(s) and the resistance is the sum of the ballast resistance and the plasma resistance. It is likely that variations in ignition behavior would be observed if changes were made to the ballast resistance and power supply output capacitance values.

As a preliminary test, the power supply current limit setting was varied to see whether the ignition behavior would be affected. The resulting waveforms are shown in Fig. 4 for ignitions that were performed with $1.5 \mathrm{~A}, 1.0 \mathrm{~A}$, and 0.5 A settings. The $0.5 \mathrm{~A}$ setting resulted in highly oscillatory behavior in which the plasma discharge between the electrodes would decouple and reform a number of times before a stable discharge was reached. The $1.0 \mathrm{~A}$ setting demonstrated similar behavior, although events of decoupling were much less prominent. The 1.5 A setting exhibited the most stable behavior and also provided the most consistent and repeatable ignition data across a range of flow rates. For the remainder of the heaterless hollow cathode ignition tests, the current limit was set to $1.5 \mathrm{~A}$. This was observed with both the Magna Power SL-1000-1.5 power supply used at NASA Glenn Research Center, and with the Sorensen XG 600-2.8 power supply used at Colorado State University. Although the 1.5 A setting resulted in the fastest and least erratic ignition behavior, the hollow cathode demonstrated stable operation at any of these current settings after steady state operating temperature was achieved. 

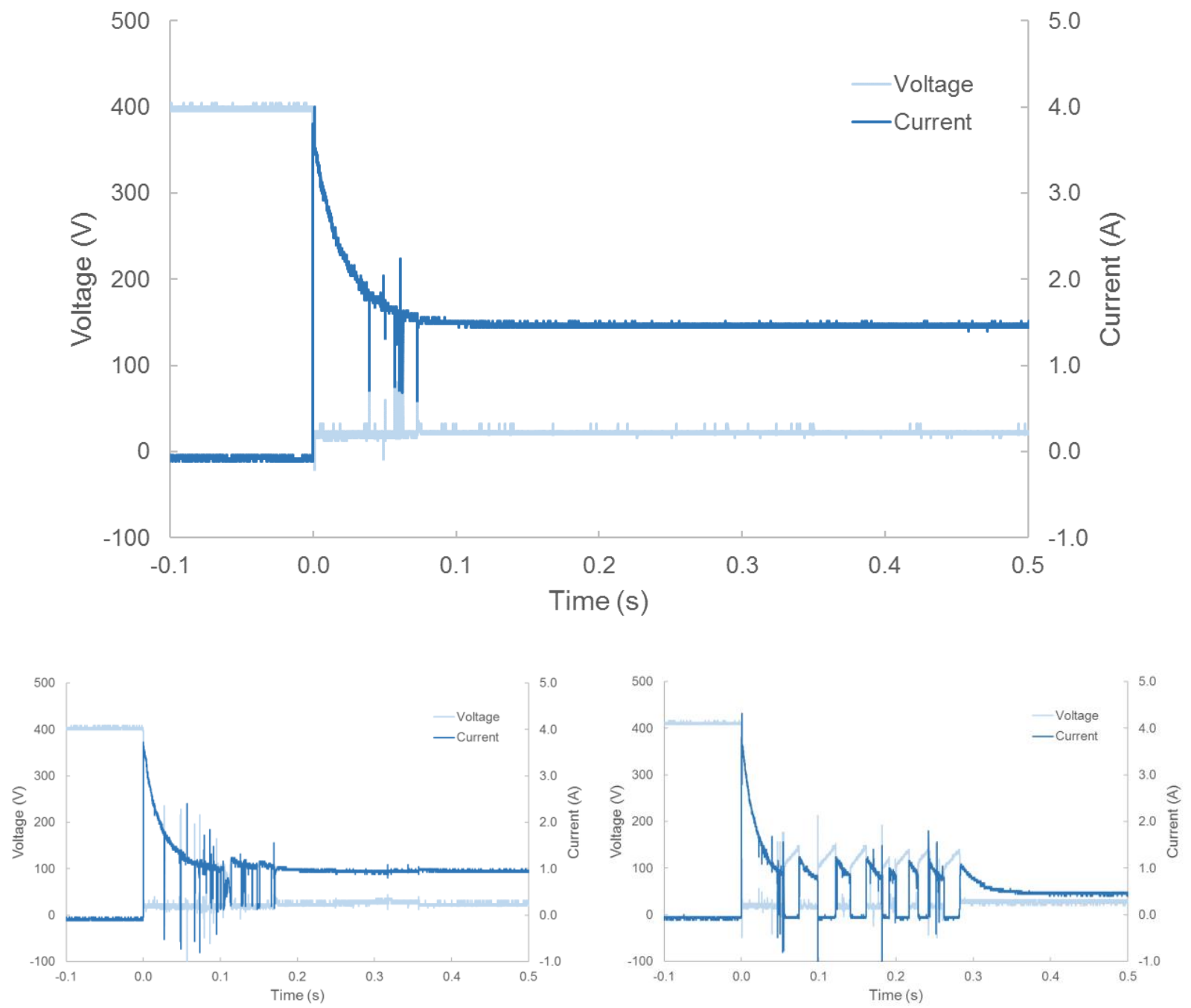

Fig. 4 Voltage and current flow between electrodes during ignition with power supply set to $1.5 \mathrm{~A}$ (top), 1.0 A (bottom-left), and $0.5 \mathrm{~A}$ (bottom-right).
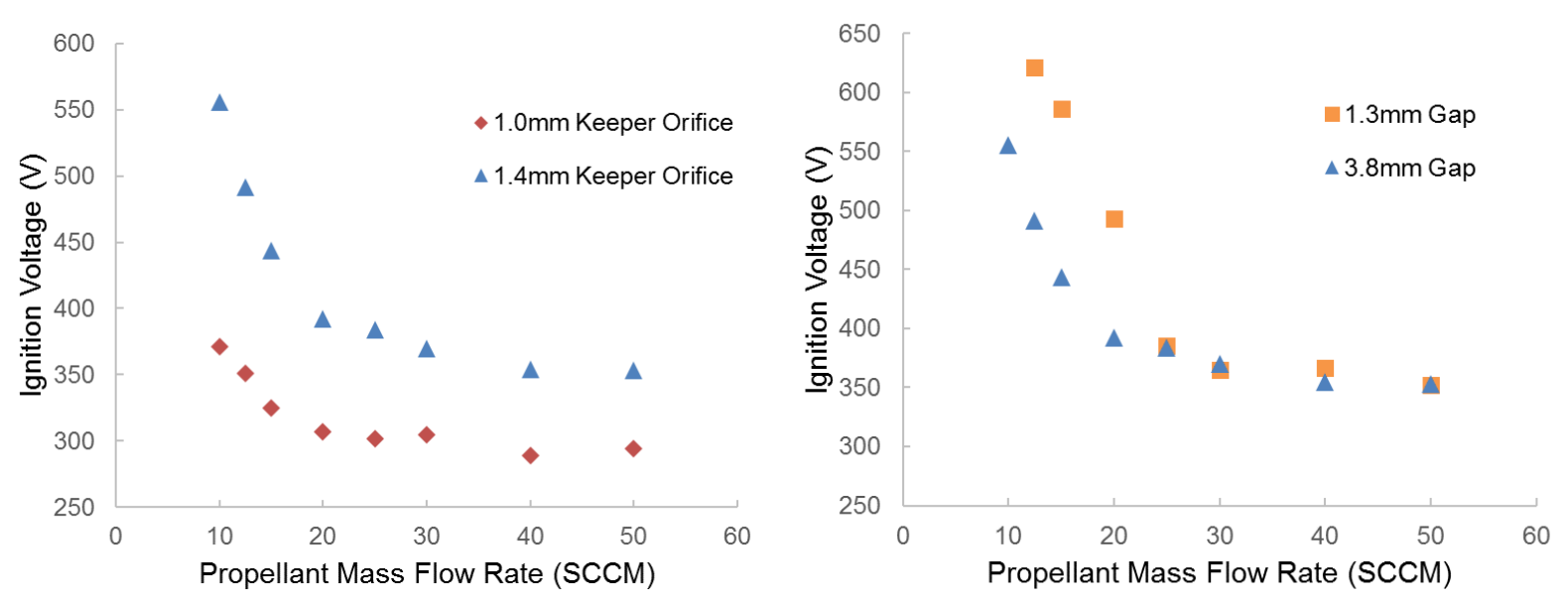

Fig. 5 Ignition voltage as a function of xenon mass flow rate for various heaterless hollow cathode configurations. 
The plots in Fig. 5 show the results of varying the keeper orifice diameter and the axial distance between the cathode and keeper electrodes. In these plots, the average ignition voltage is plotted as a function of xenon mass flow rate for each hollow cathode configuration. In all cases, a downward sloping trend is observed. This indicates that the internal gas pressure and distance between electrodes are such that these data correspond to the region of the Paschen curve that is to the left of the minimum. Fig. 5 (left) shows there is a distinct difference in ignition voltage at a given propellant mass flow rate depending on the diameter of the keeper orifice. In both of these cases, the axial distance between the cathode and keeper was approximately $3.8 \mathrm{~mm}$. The lower ignition voltages observed with the smaller keeper orifice can be attributed to the increased flow resistance and thus higher gas pressures between the cathode and keeper electrodes. Fig. 5 (right) shows ignition voltages for two different axial distances between electrodes. The keeper orifice diameter was $1.4 \mathrm{~mm}$ in both of these tests. As one would predict using Paschen's Law, the smaller distance exhibited higher ignition voltages at flow rates below $25 \mathrm{sccm}$. However, at higher flows, the two hollow cathode assemblies demonstrated similar ignition voltages. Given that these results deviate from the theory of Paschen's Law, perhaps it is false to assume that electrical breakdown occurs only in the axial direction. Due to nonuniformities in electrode distance and internal pressure, it is possible that the location of the plasma discharge formation could vary depending on the flow condition.

Immediately after an arc discharge is formed, the cathode begins to be heated by the plasma. We hypothesize that during this time, the discharge current is produced by a volumetric ionization process and that free electrons created by ionization events are emitted from the internal plasma to the keeper while the ions are neutralized at the cathode surface where they deliver kinetic and internal energy. Secondary electron emission from the surface of the cathode could also provide a portion of the discharge current. As the temperature of the cathode rises due to ion bombardment, the cathode transitions to (predominantly) thermionic emission of electron current. It is likely that the plasma potential inside the hollow cathode and the voltage between the cathode and keeper electrodes decreases as thermionic emission increases, and by monitoring the keeper voltage over time, the duration of the cathode warm up period could be approximated. An example of these data are shown in Fig. 6 where the keeper voltage is observed to reach a steadystate value within 30 seconds, indicating that the warm-up period has completed. It is likely that the duration of the warm-up period would vary depending on the size of the cathode, the ballast resistance value, and the power supply current limit, however this has not yet been investigated.

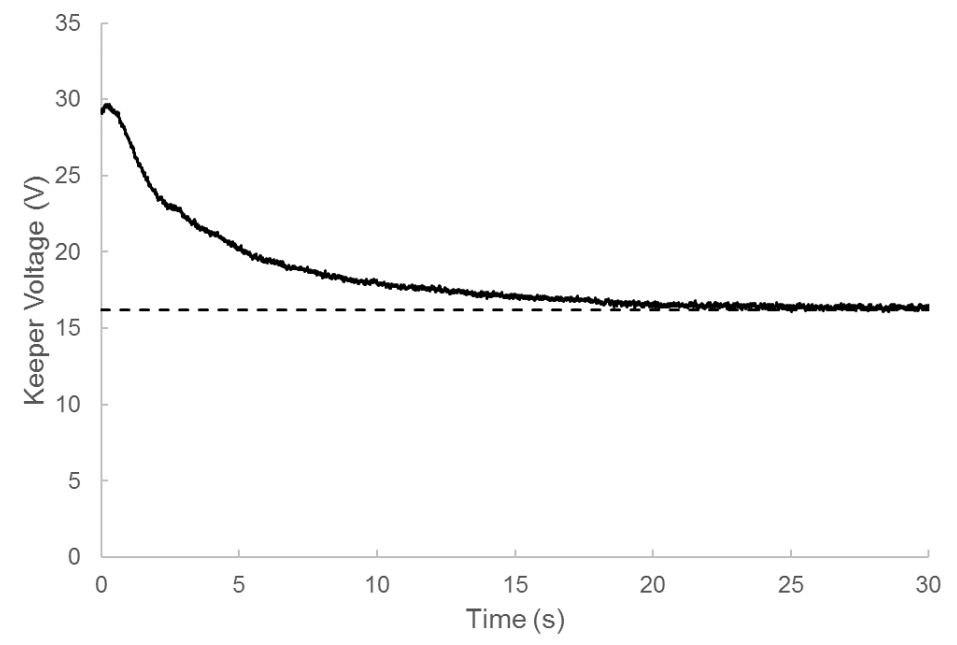

Fig. 6 Keeper voltage plotted as a function of time immediately after ignition of a heaterless hollow cathode.

\section{Discussion}

The discharge initiation experiment showed that, in accordance with Paschen's Law, the composition of the propellant gas has a profound effect on the voltage required to initiate a plasma discharge at a given gas pressure and electrode distance. This suggests that a heaterless hollow cathode assembly will likely exhibit significantly different ignition characteristics between different propellant gasses. In designing and testing heaterless hollow cathode assemblies, it may be useful to designate a unit with specified ignition characteristics as being specific to a certain propellant gas. If optimal ignition performance is desired, it would be wise to use the appropriate propellant gas during ignition testing in a cathode development activity. Additionally, it was found that the cathode material (and its surface state) does not have a significant effect on the breakdown voltage of a gas. This means that the ignition behavior of a 
hollow cathode assembly should not be greatly affected if a different cathode tube material was used. Furthermore, it was shown that both the keeper orifice diameter and the axial position of the cathode relative to the keeper have an effect on the voltage required to ignite a plasma arc discharge at a given flow rate. While these results generally align with Paschen's Law, there are some discrepancies that suggest that the inherent non-uniformities in electrode spacing and pressure within a hollow cathode assembly must be considered before one can accurately estimate the ignition voltage without resorting to experiments. A more comprehensive model of the ignition process is judged to be necessary to better predict ignition behavior.

To integrate a heaterless hollow cathode in spaceflight electric propulsion system, it will be necessary to design the propellant feed system and power processing unit (PPU) so that sufficient flow and bias conditions can be met, and a plasma arc discharge can be initiated reliably. Beyond this, it is also necessary that the plasma discharge be maintained after it is initiated. It is important to acknowledge that a typical spaceflight PPU will likely behave very differently from a laboratory power supply. For example, the laboratory power supplies used in this study have relatively large output capacitance which is used to minimize ripple in the current output. The energy stored in the output capacitor(s) is released through the circuit at the moment that a plasma discharge is formed. Evidence of this is apparent in Fig. 4 in the exponential decay of current that is characteristic of a resistor-capacitor circuit. The energy released from the capacitor during this time may actually be helpful in maintaining the plasma discharge. Additionally, a ballast resistance was added to the keeper circuit in this research to limit the current flow during the discharge of this capacitance. A typical spaceflight PPU uses a much smaller output capacitance in the keeper circuit and does not include a large ballast resistance. In developing a heaterless hollow cathode for spaceflight, this should be considered because the ignition behavior of a heaterless hollow cathode could vary dramatically between a laboratory power supply and a spaceflight PPU. Furthermore, it was shown that ignition of a heaterless hollow cathode is more reliable when a higher discharge current is maintained immediately after the plasma discharge is initiated. Considering that most PPUs are not designed to drive 1.5 A through the keeper circuit, as an alternative, it may be possible to ignite a heaterless hollow cathode with both the keeper and anode circuits actively driving current during the ignition process. To integrate a heaterless hollow cathode in an electric propulsion system, it will likely be necessary to develop a PPU in parallel with a heaterless hollow cathode so that compatibility between the two can be optimized.

\section{Future Work}

It is often assumed that the plasma discharge in a hollow cathode is initiated in a region with the highest neutral gas density, which is presumably between the cathode orifice and keeper orifice. It is possible, however, that given certain flow conditions and hollow cathode assembly geometries, that the pressure and electrode distance conditions preferable for breakdown could exist in another location. This could result in out-of-trend ignition voltages or other issues with ignition performance. A computational model of the ignition process that includes both ionization and heat transfer processes would be a very useful tool that could be used in the preliminary design of a heaterless hollow cathode. Using a well-refined model, the ignition behavior could be predicted before constructing the hollow cathode assembly and performing laboratory testing. An ignition model would incorporate the concepts discussed in this paper, such as Paschen's Law which relates the breakdown voltage to the gas pressure and the distance between electrodes. Unfortunately, translating this model to a heaterless hollow cathode assembly is not straightforward because both the electrode spacing, and internal pressure are non-uniform in a hollow cathode assembly, and the propellant flow is in a transition flow regime which is difficult to model. To create an accurate model of the ignition process, it will be necessary to model the electric field, the flow field, the electrode spacing, and finally, the ionization process. In developing such a model, it will be useful to repeat the experiments described in this paper to confirm and/or refine the accuracy of the model.

There are concerns that ignition of a plasma arc discharge in cold cathode may cause the cathode material to be eroded by bombardment of the cathode by energetic ions during ignition causing excessive localized heating, vaporization, and sputtering of the cathode material [7, 18]. This ablation process would be best studied by performing a high number of ignition cycles and observing the resulting erosion. It is likely that the electrical behavior during ignition directly affects the amount of erosion that occurs during each ignition. Because the transient voltage and current characteristics of an ignition event are dependent upon the electrical system design and the physical conditions within the hollow cathode assembly, (such as internal pressure and electrode spacing), it would perhaps be beneficial to perform two or more repeated ignition cycle experiments; one with a lower ignition voltage and an ignition current spike of low magnitude, and another with a higher ignition voltage and a larger current spike. By automating a repeated ignition sequence, 1000 or more starts could be performed in several days. Afterwards, geometric and/or compositional changes to the cathode material could be measured, and the erosion effects quantified. 


\section{Conclusion}

The purpose of this study was to determine the factors to be considered when designing a heaterless hollow cathode for spaceflight applications. To integrate a heaterless hollow cathode in an electric propulsion thruster, it will be necessary that the propellant feed system and PPU are capable of providing the flow and bias conditions that are necessary to ignite a plasma arc discharge within the hollow cathode assembly. In some cases, (especially in smallsatellite propulsion systems), it may not be feasible to implement advanced PPU and feed system hardware capable of providing high flow and/or voltage. In these cases, it will be necessary to design the heaterless hollow cathode to ignite reliably under conditions that the system hardware is capable of providing. The results that are presented in this paper help to form some guidelines for how one would approach this challenge.

\section{Acknowledgments}

The authors would like to thank Casey Farnell and Seth Thompson of Plasma Controls, LLC, for developing the heaterless hollow cathode that was used in this research. The authors would also like to thank Kevin Blake, Kevin Rahill, and James Sadey of NASA Glenn Research Center for their assistance in configuring VF-17 for heaterless hollow cathode testing. This work was supported by a NASA Space Technology Research Fellowship under grant number 80NSSC18K1156.

\section{References}

[1] Verhey, T. R., Soulas, G. C., and Mackey, J. A., "Heater Validation for the NEXT-C Hollow Cathodes," 35th International Electric Propulsion Conference, IEPC-2017-397, Atlanta, Georgia, USA, 2017.

[2] Soulas, G. C., "Status of Hollow Cathode Heater Development for the Space Station Plasma Contactor," 30th Joint Propulsion Conference, AIAA-94-3309, Indianapolis, Indiana: American Institute of Aeronautics and Astronautics, 1994.

[3] Mackey, J. A., Shastry, R., and Soulas, G., "Characterization of the NEXT hollow cathode inserts after long-duration testing," 35th International Electric Propulsion Conference, IEPC-2017-304, Atlanta, Georgia, USA, 2017.

[4] Shastry, R., and Soulas, G. C., "Post-test Inspection of NASA's Evolutionary Xenon Thruster Long-Duration Test Hardware: Discharge and Neutralizer Cathodes," 52nd Joint Propulsion Conference, Salt Lake City, UT: American Institute of Aeronautics and Astronautics, 2016.

[5] Polk, J. E., Mikellides, I. G., Capece, A. M., and Katz, I., "Barium depletion in hollow cathode emitters," Journal of Applied Physics, vol. 119(2), 2016.

[6] Rubin, B., and Williams, J. D., 2008, "Hollow cathode conditioning and discharge initiation," Journal of Applied Physics, vol. 104(5), 2008.

[7] Lev, D., and Appel, L., "Heaterless Hollow Cathode Technology - A Critical Review," Space Propulsion, SP2016_3125366, Rome, Italy, 2016.

[8] Conversano, R. W., Lobbia, R. B., Tilley, K. C., Goebel, D. M., and Reilly, S. W., "Development and Initial Performance Testing of a Low-Power Magnetically Shielded Hall Thruster with an Internally-Mounted Hollow Cathode," 35th International Electric Propulsion Conference, IEPC-2017-64, Atlanta, Georgia, USA, 2017.

[9] Vekselman, V., Krasik, Y., Gleizer, S., Gurovich, V., Warshavsky, A., and Rabinovich, L., "Characterization of a Heaterless Hollow Cathode," Journal of Propulsion and Power, vol. 29(2), 2013, pp. 475-486.

[10] Daykin-Iliopoulos, A., Gabriel, S., Golosnoy, I., Kubota, K., and Funaki, I., "Investigation of Heaterless Hollow Cathode Breakdown," 34th International Electric Propulsion Conference, IEPC-2015-193, Kobe, Japan, 2015.

[11] Hall, S. J., Sarver-Verhey, T. R., Frieman, J. D., Kamhawi, H., and Myers, J. L., "Preparation for Hollow Cathode Testing for the Advanced Electric Propulsion System at NASA Glenn Research Center," 54 ${ }^{\text {th }}$ Joint Propulsion Conference, Cincinnati, OH: American Institute of Aeronautics and Astronautics, 2018.

[12] Farnell, C. C., Farnell, C. C., Williams, J. D., Williams, D. D., Nguyen, B. G., Greiner, K. E., and Ham, R. K., “Simplified Formation Process of a Low Work Function Insert," US 10,002738, US Patent, 2018.

[13] Wittenburg, H., “Gas Tube Design,” Electron Tube Design, Lancaster, 1962, pp. 792-817.

[14] Dominguez, A., "Derivation of the Paschen curve law," ALPhA Laboratory Immersion, July 2014.

[15] Chung, M. S., Hong, S. C., Cutler, P. H., Miskovsky, N. M., Weiss, B. L., and Mayer, A., 2006, "Theoretical analysis of triple junction field emission for a type of cold cathode," Journal of Vacuum Science \& Technology, 24(909).

[16] Iwata, M., Khan, A. R., Igawa, H., Toyoda, K., Cho, M., and Fujita, T., 2012, "Development of Electron-Emitting Film for Spacecraft Charging Mitigation," Journal of Spacecraft and Rockets, 49(3).

[17] Dobretsov, L. N., Gomoyunova, M. V., "Emission Electronics," Israel Program for Scientific Translations, 1971, pp. 326336.

[18] Ning, Z., Zhang, H., Yang, L., Yu, D., "The Ignition Erosion Mechanism of Heatless Hollow Cathode," 35th International Electric Propulsion Conference, IEPC-2017-70, Atlanta, Georgia, USA, 2017. 